\title{
Role of Oxidized Mo Species on the Active Surface of Ni-Mo Electrocatalysts for Hydrogen Evolution under Alkaline Conditions
}

\author{
Jeremy A. Bau, ${ }^{\nabla}$ Sergey M. Kozlov, ${ }^{\nabla}$ Luis Miguel Azofra, Samy Ould-Chikh, Abdul-Hamid Emwas, \\ Hicham Idriss, Luigi Cavallo,* and Kazuhiro Takanabe*
}

Cite This: ACS Catal. 2020, 10, 12858-12866

Read Online

\section{ACCESS | Lill Metrics \& More | 回 Article Recommendations ｜（） Supporting Information}

ABSTRACT: A Ni-Mo composite functions as a promising non-noble metal electrocatalyst for the hydrogen evolution reaction (HER) in alkaline water. Despite its industrial relevance, the kinetic origin of the high catalytic activity remains under debate. The present report discusses a reaction mechanism of $\mathrm{HER}$ on $\mathrm{Ni}-\mathrm{Mo}$ catalysts by combining experimental and theoretical studies. In contrast to a $\mathrm{Ni}$ catalyst, a $\mathrm{Ni}-\mathrm{Mo}$ catalyst is insensitive to $\mathrm{CO}$ gas introduced during HER. In situ spectroscopic measurements including Raman spectroscopy and electron paramagnetic resonance (EPR) show that $\mathrm{Mo}^{3+}$ prevails during HER catalysis. Density functional

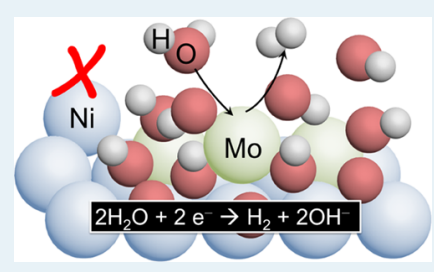
theory (DFT) simulations corroborate the thermodynamic stability and HER activity of $\mathrm{Mo}^{3+}$.

containing centers on $\mathrm{Ni}(111)$ at HER potentials. Notably, $\mathrm{Ni}$ is demonstrated to play no direct role as a catalytic site but to effectively disperse and activate the oxidized catalytic Mo species. The results illustrate how to improve the electrocatalytic activity for alkaline HER.

KEYWORDS: nickel, molybdenum, hydrogen evolution, alkaline, EPR

\section{INTRODUCTION}

Almost 25 years since Bard and Fox first defined water-based $\mathrm{H}_{2}$ generation as a "holy grail" for renewable energy, ${ }^{1}$ electrocatalytic water splitting remains a promising method for the production of $\mathrm{H}_{2}$ as fuel. This process is attractive because of the high specific energy of $\mathrm{H}_{2}$, the ubiquity of water, the ability to drive the reaction with diverse renewable power sources, and the potential for a carbon-neutral energy cycle. However, catalysts for the constituent electrocatalytic $\mathrm{H}_{2}$ evolution reaction (HER) remain dominated by noble metal catalysts, ${ }^{2-4}$ especially under acidic conditions, where a proton is a reactant and $\mathrm{M}-\mathrm{H}$ bond energies are proposed to be an effective and straightforward descriptor of catalytic activity. ${ }^{5}$ At higher $\mathrm{pH}$, reactant switching from protons to $\mathrm{H}_{2} \mathrm{O}$ as the $\mathrm{H}$ source occurs at appreciable current densities. ${ }^{6}$ Recent work on Pt-based electrocatalysts has not only advanced electrocatalytic performances but also provided insight into reaction mechanisms. For example, Markovic et al. reported on highly active $\mathrm{Ni}(\mathrm{OH})_{2} /$ Pt-based systems, ${ }^{7}$ where $\mathrm{Ni}(\mathrm{OH})_{2}$ islands on $\mathrm{Pt}$ as well as the presence of $\mathrm{Li}^{+}$through noncovalent interactions were proposed to promote the dissociation of water, thereby facilitating HER activity. ${ }^{7-9}$ Such decoration of Pt surfaces was proposed to improve HER activity by perturbing the reorganization of $\mathrm{H}_{2} \mathrm{O}$ on the Pt surface. ${ }^{10}$ Another noticeable benefit of alkaline conditions is that more earth-abundant elements can become candidates for stable electrocatalyst materials. ${ }^{11,12}$ Of these, nickel-molybdenum catalysts (Ni-Mo) exhibit excellent performance for HER under alkaline conditions when the surface area is enhanced by nanostructures. ${ }^{13-15}$ Despite being among the longest-known earth-abundant HER catalysts with good activity, the mechanism and active species of HER on $\mathrm{Ni}-\mathrm{Mo}$ are still under debate. ${ }^{16}$

Three major mechanisms have been proposed to account for the high HER activity of $\mathrm{Ni}-\mathrm{Mo},{ }^{16,17}$ namely, surface area enhancement through Mo dissolution, ${ }^{18,19}$ favorable reaction energetics at $\mathrm{Ni}_{x} \mathrm{Mo}_{y}$ alloy interfaces, ${ }^{16,20,21}$ and $\mathrm{Mo}$ as an overflow "valve" to prevent the inactivation of Ni hydride. ${ }^{22}$ All three mechanisms focus on the role of metallic $\mathrm{Ni}$ as the primary active site. In this study, with experimental and computational evidence, we present a distinct HER mechanism where Mo plays a major role in $\mathrm{Ni}-\mathrm{Mo}$ catalysts, consistent with a very recent report that emphasizes a key role of $\mathrm{MoO}_{2}$ at the $\mathrm{Ni}-\mathrm{MoO}_{2}$ interface. ${ }^{23}$ The results demonstrate that high HER activity in $\mathrm{Ni}-\mathrm{Mo}$ arises from surface-bound Mo that forms an oxo species with an unusual oxidation state $\left(\mathrm{Mo}^{3+}\right)$, which is dispersed on conductive metallic Ni. Evidence for HER-active Mo oxo species is currently found only in molecular HER catalysts, ${ }^{24-26}$ but is consistent with the report that $\mathrm{MoO}_{2}$ nanosheets (without $\mathrm{Ni}$ ) show excellent performance for alkaline HER. ${ }^{27}$ Therefore, our findings provide a consolidated mechanism for different materials as well as a link between heterogeneous and molecular HER catalysts.

Received: June 22, 2020

Revised: September 30, 2020 


\section{EXPERIMENTAL METHODS}

Electrochemistry. Electrochemical experiments were carried with a Bio-Logic SP-150 Potentiostat. The electrolyte was $0.1 \mathrm{M} \mathrm{KOH}$ with Ar bubbling (except for 99\% Ar/1\% CO for $\mathrm{CO}$ experiments). The $\mathrm{Hg} / \mathrm{HgO}$ reference electrode was calibrated with $\mathrm{Pt} / \mathrm{H}_{2}$. The counter electrode was carbon cloth. Organic experiments were performed under Ar using Schlenk techniques in $0.2 \mathrm{M} \mathrm{Bu}_{4} \mathrm{~N} \mathrm{PF} 6 . \mathrm{Bu}_{4} \mathrm{~N}-\mathrm{PF}_{6}$ was purified by recrystallization in ethanol. Purified THF was kept under a $\mathrm{K}$ mirror with a benzophenone indicator. Pt wire separated by glass frit was used as the counter electrode. $\mathrm{Ag} / \mathrm{Ag}^{+}(0.01 \mathrm{M}$ $\mathrm{AgNO}_{3} / 0.1 \mathrm{M} \mathrm{Bu}_{4} \mathrm{~N}-\mathrm{PF}_{6}$ with $\mathrm{Ag}$ wire) was used as the reference electrode. Warning: addition of water to the $\mathrm{PF}_{6}$ electrolyte can result in the formation of trace amounts of HF during electrochemical experiments. However, we were unconcerned as the experiments were short term, carried out at room temperature with limited electrochemical activity.

Preparation of Films and Materials. A Ni-Mo catalyst was deposited at $-10 \mathrm{~mA} \mathrm{~cm}{ }^{-2}$ for $40 \mathrm{~s}$ with no rotation for the flat electrode. Longer electrodeposition was conducted for $1 \mathrm{~h}$ with rotation on glassy carbon rotating disc electrodes (RDEs) at $800 \mathrm{rpm}$ or stirring on glassy carbon flat electrodes at $800 \mathrm{rpm}$. The electrodeposition was carried out in a solution of $0.25 \mathrm{M} \mathrm{Ni}\left(\mathrm{SO}_{4}\right) \cdot 6 \mathrm{H}_{2} \mathrm{O}, 0.25 \mathrm{M} \mathrm{Na}_{2} \mathrm{MoO}_{4} \cdot 2 \mathrm{H}_{2} \mathrm{O}$, and 0.30 $\mathrm{M}$ trisodium citrate adjusted to $\mathrm{pH} 10.5$ with concentrated ammonia. Before use, the glassy carbon substrates $(0.0707$ $\mathrm{cm}^{2}$ ) were polished with $1 \mu \mathrm{m}$ diamond and $0.05 \mu \mathrm{m}$ alumina, followed by washing in water and cycling in $1 \mathrm{M} \mathrm{HClO}_{4}$.

In Situ Electron Paramagnetic Resonance (EPR). A 4 $\mathrm{cm}$ length of $\mathrm{Au}$ wire was flattened, leaving the rest of the wire attached to the top of the flat area. After cleaning by polishing and electrochemical cycling in $1 \mathrm{M} \mathrm{HClO}_{4}, \mathrm{Ni}-\mathrm{Mo}$ was deposited. The air-dried electrode was assembled with a $\mathrm{Pt}$ counter electrode and a $\mathrm{Ag} / \mathrm{Ag}^{+}$reference electrode under $\mathrm{Ar}$ in a Wilmad-LabGlass flat electrolysis cell filled with $0.2 \mathrm{M}$ $\mathrm{Bu}_{4} \mathrm{NPF}_{6}$ and sealed. EPR spectra were recorded using an $\mathrm{X}$ band continuous-wave Bruker EMX PLUS spectrometer (BrukerBioSpin, Rheinstetten, Germany) equipped with a standard resonator for high-sensitivity CW-EPR operating at 9.795 GHz. The spectra were measured at a microwave attenuation of $20 \mathrm{~dB}$ with a modulation amplitude of $5 \mathrm{G}$ and a modulation frequency of $100 \mathrm{kHz}$.

In Operando Surface-Enhanced Raman Spectroscopy (SERS). Raman spectroscopy was performed on gold electrodes (area $\sim 0.5 \mathrm{~cm}^{2}$ ). The open-top cell was designed for a Horiba Aramis Raman spectrometer with an Olympus 60x water immersion lens. The lens was protected with a $0.013 \mathrm{~mm}$ Teflon film (American Durafilm). ${ }^{28}$ The electrodes were roughened by cycling in $0.1 \mathrm{M} \mathrm{KCl}^{29}$ Films were deposited from a Ni-Mo deposition with stirring at $800 \mathrm{rpm}$ at $-10 \mathrm{~mA}$ $\mathrm{cm}^{-2}$ for $2 \mathrm{~min}$. The electrolyte, $0.1 \mathrm{M} \mathrm{KOH}$, was bubbled with Ar.

Composition Measurements by Inductively Coupled Plasma Mass Spectrometry (ICP-MS). Ni-Mo deposited on a glassy carbon electrode was dissolved in small volumes (100 $\mu \mathrm{L}$ of $70 \% \mathrm{HNO}_{3}$ ). The acid was added to a $7 \mathrm{~mL}$ acid solution $\left(6 \mathrm{~mL} 70 \% \mathrm{HNO}_{3}, 1 \mathrm{~mL} 40 \% \mathrm{HF}\right)$ and digested via a microwave. Diluted samples were used for ICP-MS measurements on a PerkinElmer ELAN DRC II mass spectrometer. For dissolved Mo experiments, $1 \mathrm{~mL}$ of $0.1 \mathrm{M} \mathrm{KOH}$ was placed in a $10 \mathrm{~mL}$ beaker. Electrochemical measurements were performed as described and then the electrolyte was mixed with the $\mathrm{HNO}_{3} / \mathrm{HF}$ solution and digested before analysis.

Electron Microscopy and Energy-Dispersive X-ray (EDX) Spectroscopy. Scanning electron microscopy (SEM) was performed on an FEI Nova Nano SEM630 (5 kV accelerating voltage). High-angle annular dark-field scanning transmission electron microscopy (HAADF-STEM) was performed with a Titan Themis-Z (Thermo Fisher Scientific) at an accelerating voltage of $300 \mathrm{kV}$ with a beam current of $0.5-0.8 \mathrm{nA}$. The data were acquired with a convergence angle of $29.9 \mathrm{mrad}$ and an HAADF inner angle of $119 \mathrm{mrad}$. An Xray energy-dispersive spectrometer (FEI SuperX, $\approx 0.7$ sR collection angle) was also utilized in conjunction with DFSTEM imaging to acquire STEM-EDX spectrum-imaging data sets (image size: $1024 \times 1024$ pixels, dwell time $5 \mu \mathrm{s}$ ). During the acquisition, a corresponding EDX spectrum was acquired at every image pixel to generate the elemental maps of Mo and Ni atoms simultaneously. Spectrum-imaging data sets were acquired in frame mode, in which an electron beam was allowed to dwell at each pixel for only a few microseconds to maintain a total frame time of $6.5 \mathrm{~s}$ or less. All data sets were acquired and analyzed with Velox (Thermo Fisher Scientific). The elemental maps for $\mathrm{Mo}, \mathrm{Ni}$, and $\mathrm{O}$ atoms were computed using their respective $\mathrm{K} \alpha$ lines after background subtraction. The generated maps were slightly post-filtered applying a Gaussian filter $(\sigma=0.5)$.

\section{COMPUTATIONAL DETAILS}

Spin-polarized density functional calculations were performed with VASP. ${ }^{30}$ Simulations of electrochemical processes were performed using the rPBE exchange-correlation functional. ${ }^{31}$ The core electrons were described using projector-augmented wave (PAW) pseudopotentials, $^{32}$ whereas valence electrons were described using a plane-wave basis set with a cutoff energy of $400 \mathrm{eV}$. Hubbard $U$ corrections ${ }^{33}$ of $2 \mathrm{eV}$ were applied to d-orbitals of Mo according to the method proposed by Dudarev et al. to ensure electron localization in $\mathrm{MoO}_{x} \mathrm{H}_{y}$ clusters. The dispersive interactions were taken into account using the D3 method by Grimme et al. ${ }^{34}$ Finally, the effect of solvent on the electrochemical processes was calculated using VASPSol. ${ }^{35}$ During self-consistent field calculations, each system was allowed to change its spin configuration to achieve the lowest energy. The reciprocal space was sampled by a $5 \times 5$ $\times 1 k$-point grid in 2D slab calculations, whereas unsupported gas-phase species were calculated using only the $\Gamma$-point in reciprocal space. For the $\mathrm{MoO}_{x}$ crystal, the number of $k$-points was chosen in line with the guidelines of the Materials Project. First-order Methfessel-Paxton smearing of $0.2 \mathrm{eV}$ was applied to the electronic occupation numbers. The geometry optimization of intermediates was performed until the forces on each atom were below $0.2 \mathrm{eV} \mathrm{nm}^{-1}$.

The $\mathrm{Ni}(111)$ surface was modeled by a three-layer-thick $\mathrm{p}(4$ $\times$ 4) slab with dimensions of $997 \mathrm{pm} \times 997 \mathrm{pm}$. This yielded binding energies of Mo clusters that were different by only 0.01 $\mathrm{eV}$ from those calculated on a similar eight-layer slab. The separation between adjacent slabs exceeded $1.5 \mathrm{~nm}$. CO adsorption energies were calculated as $E_{\text {ads }}=E(\mathrm{CO})+$ $E$ (substrate) $-E(\mathrm{CO} /$ substrate $)$, where $E(X)$ indicates the density functional theory (DFT) energy of either CO, substrate, or the adsorption complex. According to this definition, positive values indicate stronger binding.

The relative Gibbs energies of supported $\mathrm{MoO}_{x} \mathrm{H}_{y}$ clusters were calculated as 


$$
\begin{aligned}
G_{\mathrm{rel}}= & G\left(\mathrm{MoO}_{x} \mathrm{H}_{y}\right)-G(\mathrm{Mo})-x \times G_{\mathrm{eff}}(\mathrm{O}) \\
& -y / 2 \times G\left(\mathrm{H}_{2}\right)+(y-2 x) \times q U
\end{aligned}
$$

where $G\left(\mathrm{MoO}_{x} \mathrm{H}_{y}\right)$ and $G(\mathrm{Mo})$ are the Gibbs energies of a supported $\mathrm{MoO}_{x} \mathrm{H}_{y}$ cluster and supported $\mathrm{Mo}$ atom on a $\mathrm{Ni}(111)$ surface, respectively. $G_{\text {eff }}(\mathrm{O})=G\left(\mathrm{H}_{2} \mathrm{O}\right)-G\left(\mathrm{H}_{2}\right)$ is the effective Gibbs energy of $\mathrm{O}$ atoms in the electrochemical system at the reversible hydrogen electrode (RHE) electrode potential, and $G\left(\mathrm{H}_{2} \mathrm{O}\right)$ and $G\left(\mathrm{H}_{2}\right)$ are the Gibbs energies of water and hydrogen molecules, respectively. Finally, $q$ is the atomic unit of charge and $U$ is the electrode potential with respect to RHE. The relative Gibbs energy of the $\mathrm{MoO}_{x} \mathrm{H}_{y}$ cluster defined in such a way reflects the reaction energy of the cluster formation from a Mo adatom on the $\mathrm{Ni}(111)$ surface and $\mathrm{O}$ and $\mathrm{H}$ species derived from water

$$
\begin{aligned}
& \mathrm{Mo} / \mathrm{Ni}+(y-x) \mathrm{H}_{2} \mathrm{O}+(y-2 x) \mathrm{e}^{-} \\
& \rightarrow \mathrm{MoO} x \mathrm{H}_{y} / \mathrm{Ni}+(y-2 x) \mathrm{OH}^{-}
\end{aligned}
$$

We performed a systematic screening and identified 37 cluster compositions that do not decompose during geometry optimization. For these clusters, we investigated various types of coordination to the $\mathrm{Ni}(111)$ surface, e.g., with one $\mathrm{O}$ and one $\mathrm{OH}$ group or with a $\mathrm{H}$ atom and a Mo atom bound to the $\mathrm{Ni}(111)$ surface. For the most stable configurations, we also considered binding to various surface sites, e.g., face-centered cubic (fcc), bridge, ontop, and more sophisticated configurations for more complex adsorption modes (Figure S1). HER overpotentials $\eta$ were calculated as the absolute value of the electrode potentials vs RHE, at which both alkaline Volmer and alkaline Heyrovsky steps in HER become exothermic. Namely, $\eta=\max \left(\Delta G_{\mathrm{T}}, \Delta G_{\mathrm{H}}\right)$, where $\Delta G_{\mathrm{T}}$ and $\Delta G_{\mathrm{H}}$ are the calculated reaction Gibbs free energies of alkaline Tafel and Heyrovsky steps, respectively, calculated at the RHE potential. The Gibbs energies were calculated at $293 \mathrm{~K}$ and included contributions from vibrational degrees of freedom and zeropoint vibrations using harmonic approximation. Molecular vibrations were calculated using the finite difference method by displacing all atoms except $\mathrm{Ni}$ in three Cartesian directions by $\pm 3 \mathrm{pm}$. For $\mathrm{H}_{2}$ and $\mathrm{H}_{2} \mathrm{O}$ molecules, the contributions of rotational and translations degrees of freedom were also included in the Gibbs energy calculations using an ideal gas approximation at pressures of $p\left(\mathrm{H}_{2}\right)=100000 \mathrm{~Pa}$ and $p\left(\mathrm{H}_{2} \mathrm{O}\right)$ $=3534 \mathrm{~Pa}$. Whereas the former pressure originates from the definition of a reversible hydrogen electrode potential, the latter value is the pressure of water vapors at room temperature. Under this pressure, Gibbs energies of water molecules in the gas phase and the liquid phase are equal, which allows us to estimate the Gibbs energy of molecules of liquid water participating in the electrochemical reactions.

\section{RESULTS AND DISCUSSION}

Visibly flat films of $\mathrm{Ni}-\mathrm{Mo}$ were prepared through citrate electrodeposition at cathodic current. ${ }^{13,36}$ Co electrodeposition of $\mathrm{Ni}$ and Mo for $30 \mathrm{~s}$ results in an onset potential for HER close to the thermodynamic HER potential of $0 \mathrm{~V}$ vs HER. As previously demonstrated, ${ }^{37}$ electrocatalyst surface area enhanced by longer electrodeposition has a strong impact on the HER current based on the geometric surface area. By increasing the deposition time from $30 \mathrm{~s}$ to $1 \mathrm{~h}$, the overpotential $(\eta)$ to reach $-10 \mathrm{~mA} \mathrm{~cm} \mathrm{~cm}^{-2}$ was reducedfrom 280 to $95 \mathrm{mV}$ in $0.1 \mathrm{M} \mathrm{KOH}$, a performance comparable to that of the state-of-the-art Ni-Mo. ${ }^{16,17}$ Therefore, the estimated intrinsic activity (exchange current density) of $\mathrm{Ni}-$ Mo is not very high compared to, e.g., Pt but can be increased by improving the active surface area. Under this electrodeposition protocol, a final Ni/Mo ratio of approximately 4:1 was deposited regardless of the deposition time, confirmed by inductively coupled plasma mass spectrometry (ICP-MS), consistent with the literature. ${ }^{38}$ Scanning electron microscopy (SEM) of the sample for $1 \mathrm{~h}$ deposition revealed that the deposition resulted in the formation of nanoparticulate features of $<10 \mathrm{~nm}$, accumulating on a three-dimensional structure (Figure $1 \mathrm{~b}$ ). The electrodes were fragmented and examined

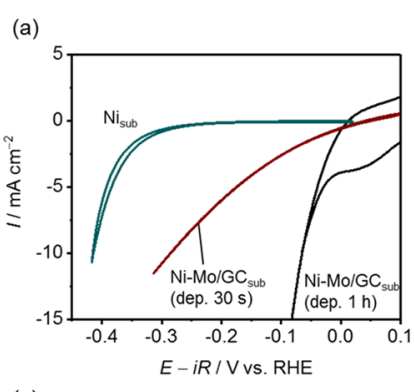

(b)

(c)

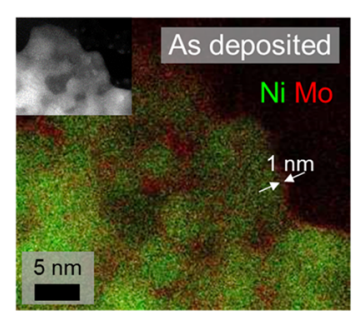

(d)
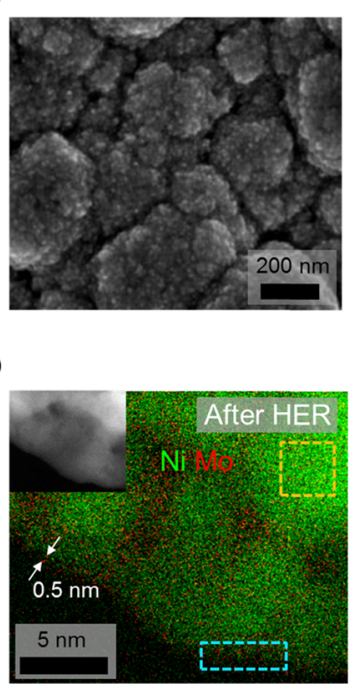

Figure 1. (a) Cyclic voltammograms of Ni-Mo deposited on glassy carbon (GC) for $30 \mathrm{~s}$ or $1 \mathrm{~h}$, and $\mathrm{Ni}_{\text {sub }}$ (a flat $\mathrm{Ni} \mathrm{RDE}$ ) only. Measurements carried out in $0.1 \mathrm{M} \mathrm{KOH}$ with bubbling Ar, scan rate: $100 \mathrm{mV} \mathrm{s}^{-1}$. (b) SEM image of a Ni-Mo electrode for $\mathrm{Ni}-\mathrm{Mo} / \mathrm{GC}$ deposited for $1 \mathrm{~h}$, EDX maps of a fragmented $\mathrm{Ni}-\mathrm{Mo}$ electrode, (c) freshly deposited, and (d) after HER testing. Comparative EDX spectra for the edge (cyan) and interior (yellow) regions are shown in the Supporting Information. Inset: HAADF-STEM of the catalyst.

using high-angle annular dark-field scanning transmission electron microscopy (HAADF-STEM) and energy-dispersive $\mathrm{X}$-ray (EDX) spectroscopy before (as deposited) and after HER operation $\left(-10 \mathrm{~mA} \mathrm{~cm}{ }^{-2}, 1 \mathrm{~h}\right)$. While electrodeposited $\mathrm{Ni}-\mathrm{Mo}$ is typically assumed to be a well-mixed alloy, ${ }^{20}$ closer examination of the material revealed the formation of a network of interconnected $\mathrm{Ni}$ or $\mathrm{Ni}_{x} \mathrm{Mo}_{y}$ nanoparticles $(\sim 5$ to $10 \mathrm{~nm}$ ) covered by thin layers of Mo oxide, at least under the conditions for STEM observation after the HER test. The enhanced presence of Mo on the surface of the nanoparticles was confirmed by comparisons of the EDX spectra between the core and edges of the nanoparticles (Figure S2). The Mo layers were thicker $(\geq 1 \mathrm{~nm})$ in freshly deposited samples, indicating that excess Mo was etched during the HER (Figure 1c,d). However, the outermost surface consisting of Mo in direct contact with $\mathrm{Ni}$ or $\mathrm{Ni}_{x} \mathrm{Mo}_{y}$ has the potential of providing unique catalytic and material properties for HER. Despite the potential presence of segregated phases, only fcc $\mathrm{Ni}$ was observed during electron diffraction measurements (Figure S3).

To provide additional insight into the active species during $\mathrm{HER}$, an electrochemical measurement in the presence of $\mathrm{CO}$ 
was conducted to see whether the catalyst was poisoned as commonly found for some metallic catalysts. As can be seen from Figure $2 \mathrm{a}, \mathrm{Ni}-\mathrm{Mo}$ was found to be almost completely
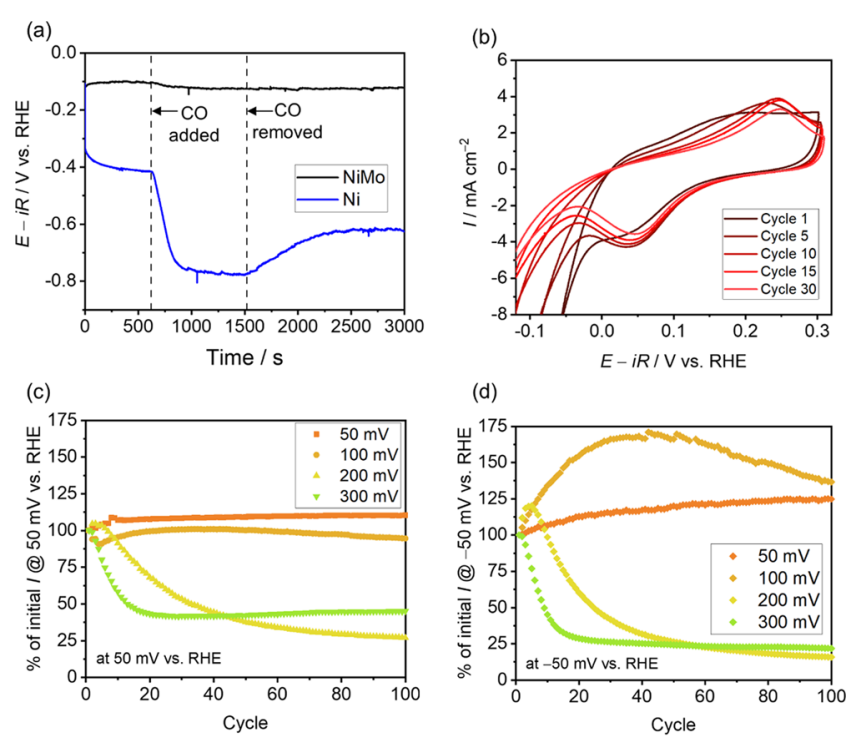

(e)

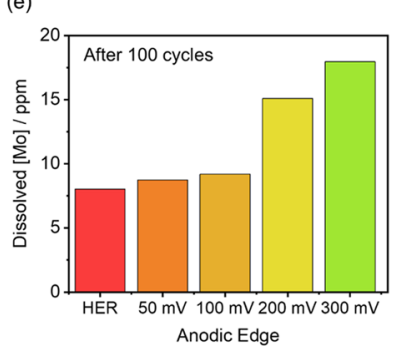

Figure 2. (a) Chronopotentiometry on $\mathrm{Ni}-\mathrm{Mo}$ and $\mathrm{Ni}_{\text {sub }}$ electrodes at $-10 \mathrm{mV} \mathrm{cm}^{-2}$ in $1 \% \mathrm{CO} / \mathrm{Ar}$ (the initial and final gas is $\mathrm{Ar}$ ), (b) Cyclic voltammograms of $\mathrm{Ni}-\mathrm{Mo}$ electrodes cycled to $300 \mathrm{mV}$ vs RHE after 1, 5, 10, 15, and 30 cycles. (c) Cathodic current density (HER activity) at $-50 \mathrm{mV}$ vs RHE after each cycle to each respective anodic edge. (d) Remaining Mo capacitance (measured by anodic current at $50 \mathrm{mV}$ vs RHE) after each cycle to each respective anodic edge. (e) ICP-MS measurements of dissolved Mo after 100 cycles to each anodic edge. The experimental setup is described in the Experimental Methods section.

immune to $\mathrm{CO}$ poisoning in contrast to Ni. Ordinarily, $\mathrm{CO}$ binds metallic $\mathrm{Ni}$ surface sites under cathodic potentials, drastically increasing the potential needed to drive the HER. $^{39,40}$ DFT calculations also demonstrated that this immunity did not arise from subsurface Mo affecting the binding affinity of $\mathrm{Ni}$ to $\mathrm{CO}$ through electronic or other effects (see the Supporting Information). In contrast to previous consensus views on $\mathrm{Ni}-\mathrm{Mo}$ surfaces responsible for the $\mathrm{HER},{ }^{16,19}$ these results are consistent with the mechanism where metallic $\mathrm{Ni}$ is not directly involved as a site for hydrogen binding in HER catalysis as the DFT calculation also confirms. It is therefore reasonable to consider that the surface catalytic sites do not exhibit a metallic character, i.e., Ni metal or $\mathrm{Ni}_{x} \mathrm{Mo}_{y}$ metallic alloy forming metal hydride species. Alternatively, since surface $\mathrm{Ni}(\mathrm{OH})_{2}$ seems to be automatically present on $\mathrm{Ni}_{x} \mathrm{Mo}_{y}$ surfaces, ${ }^{16} \mathrm{Ni}(\mathrm{OH})_{2}$ might serve as a cocatalyst for the HER under alkaline conditions. ${ }^{7}$ We thus examined some potential contributions from $\mathrm{Ni}(\mathrm{OH})_{2}$ reported in the literature. In the case of the formation of
$\mathrm{Ni}(\mathrm{OH})_{2}$ in $\mathrm{Ni}(\mathrm{OH})_{2} / \mathrm{Pt}$ systems, the catalytic activity for HER in $0.1 \mathrm{M} \mathrm{KOH}$ was compared with or without the addition of $1 \mathrm{mM} \mathrm{LiOH}$ (Figure S4). Since a $\mathrm{Li}$ ion is effective in assisting in the disruption and breakdown of $\mathrm{H}_{2} \mathrm{O}$ and adsorbed $\mathrm{OH}$ coordination spheres for the Volmer step as a rate-determining step, ${ }^{41}$ the use of $\mathrm{Li}^{+}$-containing electrolyte results in improved HER activity under alkaline conditions compared to $\mathrm{KOH}$ when $\mathrm{Ni}(\mathrm{OH})_{2}$ is acting as a cocatalyst on $\mathrm{Pt}^{7}$ However, no such improvement was found during the HER on $\mathrm{Ni}-\mathrm{Mo}$, leading to the conclusion that the reason for high catalytic activity is different from that of $\mathrm{Ni}(\mathrm{OH})_{2} / \mathrm{Pt}$ proposed in the literature. ${ }^{41}$ As a result of these findings, it is highly possible that Mo species function as chemisorption sites and are directly involved in the HER in $\mathrm{Ni}-\mathrm{Mo}$. In what follows, we investigated the impact of how the presence of surface Mo species affects HER performance.

The electrochemical behavior of the $\mathrm{Ni}-\mathrm{Mo}$ electrodes under alkaline conditions was directly studied by etching Mo under mild anodic conditions where Mo (but not $\mathrm{Ni}$ ) dissolution takes place. Previously, Csernica et al. ${ }^{42}$ correlated the observation of a characteristic pseudocapacitance in $\mathrm{Ni}-$ Mo nanoparticle electrodes at potentials near $0 \mathrm{~V}$ vs RHE in the presence of Mo. This pseudocapacitive behavior was confirmed with the current electrodeposited $\mathrm{Ni}-\mathrm{Mo}$ catalyst in the precatalytic potential range (Figure $2 b$ ), which could be increasingly eroded by repeated cycling to progressively more anodic potentials (200-300 $\mathrm{mV}$ vs RHE, Figure $2 \mathrm{c}$ ). When the anodic edge was $100 \mathrm{mV}$ or less, however, the pseudocapacitive behavior was preserved. Simultaneously, the loss of pseudocapacitance was concurrent with a loss in HER activity (Figure 2d). Ni-Mo electrodes cycled to potentials to $300 \mathrm{mV}$ vs RHE ultimately lost $\sim 70 \%$ of HER activity within 20 cycles. In contrast, activity for the electrodes cycled to fewer anodic edges $(50,100 \mathrm{mV}$ vs RHE) showed little loss or even a slight improvement likely associated with the reconstruction of active surfaces as a consequence of redox cycles. Therefore, using Mo capacitive behavior as an indicator of the presence of Mo, it was observed that the presence of Mo is necessary for high HER activity. To confirm this finding, dissolved Mo was measured in the electrolyte after these anodic cycling procedures were carried out. Indeed, Mo dissolution coincided with the activity loss as increased concentrations of dissolved Mo were found in the $\mathrm{KOH}$ electrolyte from $\mathrm{Ni}-\mathrm{Mo}$ cycled to anodic edges $\geq 200 \mathrm{mV}$ vs RHE as compared to those cycled to $\leq 100 \mathrm{mV}$ vs RHE (Figure 2e). In these same measurements, the solution concentration of dissolved $\mathrm{Ni}$ remained unchanged, demonstrating that the loss of Mo was independent of any hypothetical structural destabilization of the catalyst (Figure S5). The loss of Mo during repeated cycling to $300 \mathrm{mV}$ vs RHE was associated with an anodic 100 $\mathrm{mV}$ shift in the oxidation peak ${ }^{43}$ from $150 \mathrm{mV}$ vs RHE to 250 $\mathrm{mV}$ vs RHE, even as the corresponding reduction peak remained unchanged.

Having demonstrated a need for Mo species to remain on the surface of a Ni-Mo catalyst to achieve high catalytic activity, the nature of the Mo catalytic site itself under operating conditions was studied. Using operando surfaceenhanced Raman spectroscopy (SERS), fully oxidized $\mathrm{Mo}^{6+}$ species observed under an open-circuit potential disappeared under HER conditions and were replaced by a more reduced Mo oxo species, based on the work of Hardcastle and Wachs (Figure 3a). ${ }^{44}$ The exact oxidation state of Mo species cannot be determined using Raman spectroscopy alone, ${ }^{45}$ but the 
(a)

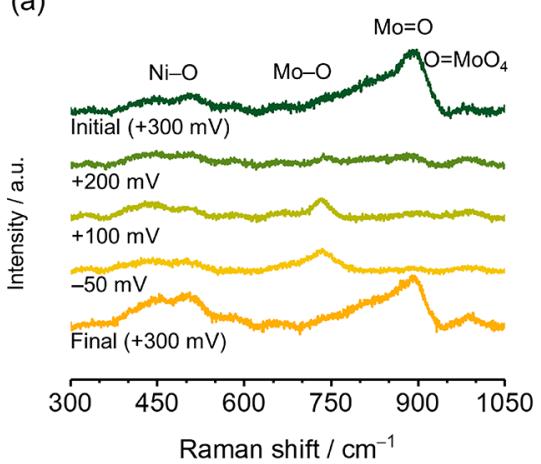

(c)

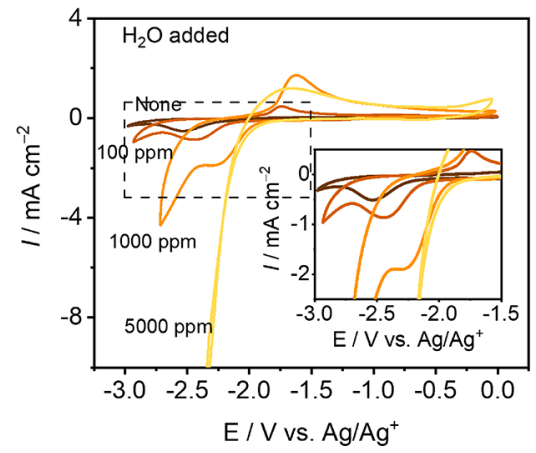

(b)

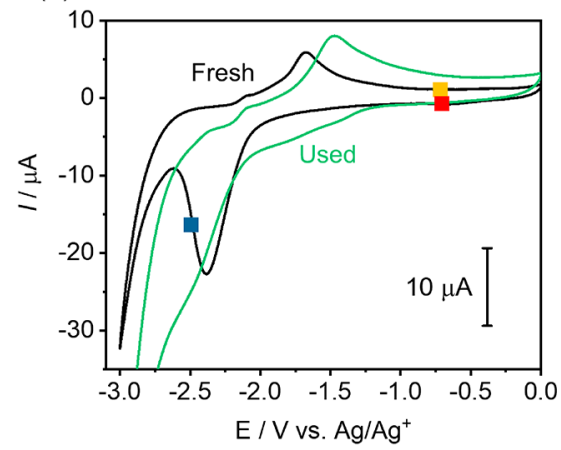

(d)

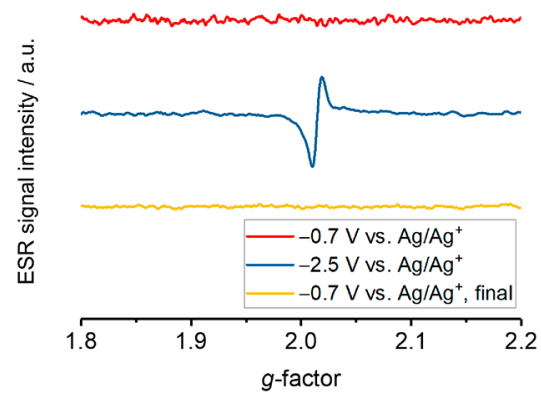

Figure 3. (a) Operando SERS of $\mathrm{Ni}_{x} \mathrm{Mo}_{y}$ deposited on a gold electrode under potentials close to the RHE potential, (b) cyclic voltammograms of fresh and used $\mathrm{Ni}_{x} \mathrm{Mo}_{y}$ electrodes in $0.2 \mathrm{M} \mathrm{Bu}_{4} \mathrm{~N} \mathrm{PF}_{6}$ in THF, (c) cyclic voltammograms of $\mathrm{Ni}_{x} \mathrm{Mo}_{y}$ electrodes after the addition of increasing amounts of water, and (d) EPR spectra of $\mathrm{Ni}_{x} \mathrm{Mo}_{y}$ electrodes held at the respective potentials in an organic electrolyte. Spectral colors match the square dots indicated in (b).

existence of Mo oxo species during the HER has been observed and modeled in molecular Mo catalysts. ${ }^{24-26}$

To differentiate between the redox behavior with and without HER catalysis, anhydrous, air-free conditions were used to study and isolate dynamic and easily oxidized active sites, as reported for a $\mathrm{Ni}-\mathrm{Fe}$ electrode for an oxygen evolution reaction. ${ }^{46} \mathrm{Ni}_{x} \mathrm{Mo}_{y}$ was found to be electrochemically responsive in a tetrahydrofuran (THF) electrolyte, exhibiting a single set of redox peaks centered at $-2.1 \mathrm{~V}$ vs $\mathrm{Ag} / \mathrm{Ag}^{+}$for both the as-prepared catalyst (Figure $3 \mathrm{~b}$ ) and the catalyst after an HER test $\left(-10 \mathrm{~mA} \mathrm{~cm}^{-2}, 0.1 \mathrm{M} \mathrm{KOH}, 1 \mathrm{~h}\right)$. To demonstrate the relevance of these redox peaks to the $\mathrm{HER}^{47}$ a small aliquot $(2.5 \mu \mathrm{L}, 100 \mathrm{ppm})$ of water was added into the THF electrolyte and voltammetry was repeated, resulting in the emergence of a catalytic wave immediately superimposable on the reduction peak (Figure 3c). The successive addition of water $(25 \mu \mathrm{L}$ total, $1000 \mathrm{ppm}$, and 125 $\mu \mathrm{L}$ total, $5000 \mathrm{ppm}$ ) eventually led to the subsuming of the reduction peak into the catalytic wave as well as an increase in the intensity of both the oxidation and reduction peaks. This redox couple is attributable to the oxidation and reduction of a Mo-charged species that is a prerequisite for the HER. This reducible Mo represented $\sim 1 \%$ of the total Mo in the electrode based on coulometric measurements of the reduction peak compared to measurements of the whole electrode composition. Considering the thickness of the entire catalyst layer ( $\sim 700 \mathrm{~nm}$ as determined via profilometry) and the minimal amount of exposed Mo on the surface of the electrode, the amount of reducible Mo is reasonable for a catalytically active surface species. Most Mo present as a bulk alloy would not be electrochemically responsive.
To identify the redox peaks observed under anhydrous conditions, in operando solid-state EPR was performed. $\mathrm{Ni}_{x} \mathrm{Mo}_{y}$ was deposited on a flattened $\mathrm{Au}$ wire and placed within a flat quartz EPR cell. Afterward, the cell was filled with a THF electrolyte and sealed under Ar with reference and counter electrodes. No initial signal was detected at the opencircuit voltage $\left(\sim-0.7 \mathrm{~V}\right.$ vs $\left.\mathrm{Ag} / \mathrm{Ag}^{+}\right)$, but a lone axial signal $\left(g_{\perp}\right)$ centered at a g-factor of 2.014 was observed upon application of a potential equivalent to the reduction peak $\left(-2.5 \mathrm{~V}\right.$ vs $\mathrm{Ag} / \mathrm{Ag}^{+}$, Figure 3d). Previous work on $\mathrm{Mo}^{3+}$ containing oxides $^{48}$ and sulfates, ${ }^{49}$ as well as $\mathrm{Mo}^{3+}$-centered molecular complexes, ${ }^{26}$ demonstrates this value to be related to the presence of $\mathrm{Mo}^{3+}$. This signal cannot be ascribed to the presence of a diamagnetic species such as $\mathrm{Mo}^{4+}$. Simultaneously, $\mathrm{Mo}^{5+}$-oxide signals, characterized in particular by a $g_{\perp}$ $=1.92$, were also not detected, unlike the case of amorphous $\mathrm{MoS}_{x}{ }^{50}$ Regarding the possibility of $\mathrm{Ni}$ involvement in the EPR signal, both $\mathrm{Ni}^{2+}$ and $\mathrm{Ni}^{0}$ are EPR silent, and the $\mathrm{Ni}^{+}$ signal may appear at $g=2.1,{ }^{51}$ substantially different from the obtained signal. Therefore, it is logical to consider the presence of $\mathrm{Mo}^{3+}$ during HER. Finally, the axial nature of the signal itself reflects the coordination of $\mathrm{Mo}^{3+}$ as a surface-bound molecular species. ${ }^{52}$ The $\mathrm{Mo}^{3+}$ peak disappeared when the applied potential of the system was returned to $-0.7 \mathrm{~V}$ vs $\mathrm{Ag} / \mathrm{Ag}^{+}$, which corresponds to the oxidation peak observed during organic cyclic voltammetry. The lack of an EPR signal at this potential confirms that the Mo oxidation state under opencircuit conditions is diamagnetic and likely a low-spin configuration of $\mathrm{Mo}^{4+}$. This in operando observation of $\mathrm{Mo}^{3+}$ formation has not been reported for the heterogeneous catalysis. 
DFT calculations were used to validate the hypothesis that a surface- oxidized Mo species is responsible for the high HER activity of $\mathrm{Ni}-\mathrm{Mo}$, rather than a metallic $\mathrm{Ni}_{x} \mathrm{Mo}_{y}$ alloy. First, we calculated the thermodynamic stability and element configuration of $\mathrm{Ni}_{x} \mathrm{Mo}_{y}$ alloy nanoparticles that successfully reflect the active $\mathrm{Ni}_{x} \mathrm{Mo}_{y}$ particle size observed in the TEM image (Figure 1). From the calculation, it was found that $\mathrm{Ni}_{x} \mathrm{Mo}_{y}$ nanoalloys have solely $\mathrm{Ni}$ atoms exposed on their surface and, as a result, bind CO molecules as strongly as pure $\mathrm{Ni}$ (Figure S6). The higher calculated average $\mathrm{CO}$ binding energy of $\sim 1.31 \mathrm{eV}$ on the $\mathrm{Ni}_{x} \mathrm{Mo}_{y}$ alloy compared to $\sim 1.26$ $\mathrm{eV}$ on pure $\mathrm{Ni}$, together with the experimental evidence of $\mathrm{CO}$ insensitivity (Figure 2a; see a detailed description of DFT in the Supporting Information), proves that the $\mathrm{Ni}_{x} \mathrm{Mo}_{y}$ alloy cannot be the active phase of the synthesized catalyst.

As a feasible alternative, we considered HER on oxidized Mo species on a Ni surface. First, the thermodynamic stability of Mo species upon applied potential (Pourbaix diagram for Mo) was thoroughly investigated. The results are shown in Figures 4 and S7. The simulations also point to the existence of $\mathrm{Mo}^{3+}$

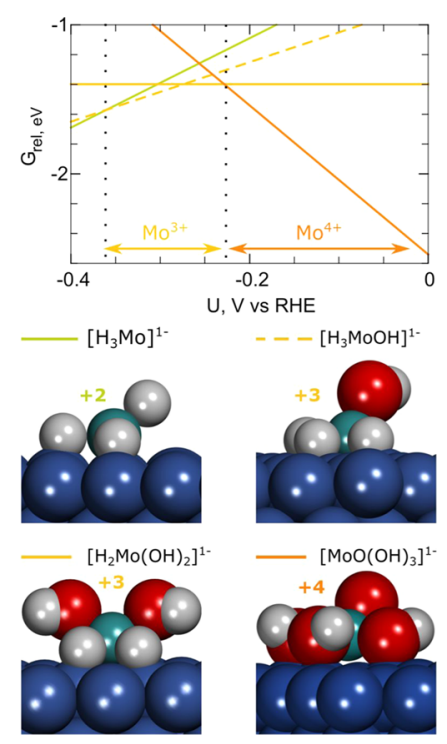

Figure 4. Relative Gibbs energies and structures of the most stable $\mathrm{H}_{x} \mathrm{MoO}_{y}(\mathrm{OH})_{z}$ clusters on the $\mathrm{Ni}(111)$ surface according to the electrode potential. The assigned formal charge on the Mo atom is given next to each structure (gray: hydrogen; green: molybdenum; red: oxygen; and blue: $\mathrm{Ni}$ ).

centers supported on Ni surfaces below the RHE potential. Because generating realistic models of amorphous Mo films on $\mathrm{Ni}$ surfaces is not straightforward, we have limited our simulations to a systematic screening of $\mathrm{H}_{x} \mathrm{MoO}_{y}(\mathrm{OH})_{z}$ clusters supported on a $\mathrm{Ni}(111)$ surface (Figure S7). The formal charges on Mo atoms in supported $\mathrm{H}_{x} \mathrm{MoO}_{y}(\mathrm{OH})_{z}$ clusters were determined by comparing their Bader charges to those on Mo atoms of unsupported clusters and $\mathrm{H}_{x} \mathrm{MoO}_{y}(\mathrm{OH})_{z}$ crystals. The Bader charge analysis ${ }^{53}$ has a certain degree of accuracy because the degree of covalence/ ionicity of bond is not a fixed number but changes with the nature of the interaction, size, and even the method of interrogation among other factors. Details of how we have approached the charge on Mo are given in Tables S1, S2 and Figures S8, S9 of the Supporting Information. In Figures 4 and 5 , the oxidation state of Mo was determined by this

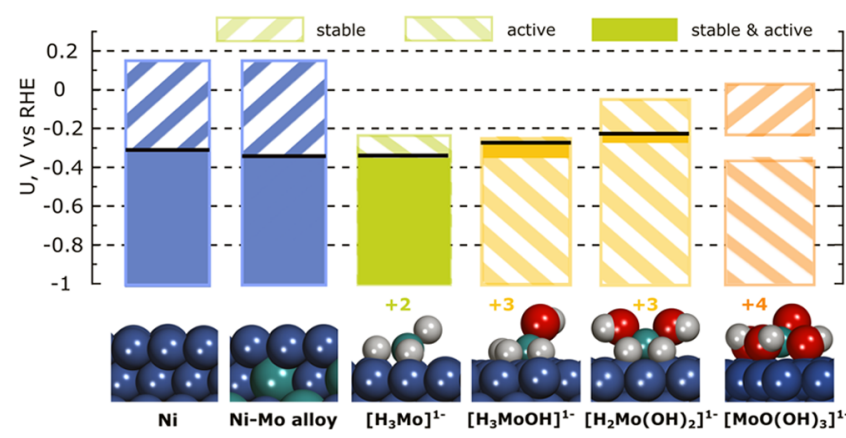

Figure 5. Overlap between the electrochemical conditions required for the stability (stripes inclined right) and HER activity (stripes inclined left) of $\mathrm{H}_{x} \mathrm{MoO}_{y}(\mathrm{OH})_{z}$ clusters. The HER overpotentials calculated on $\mathrm{Ni}$ and $\mathrm{Ni}-\mathrm{Mo}$ alloy catalysts are shown for reference; their stability under high $\mathrm{pH}$ was measured experimentally.

methodology. For example, we attributed a Mo cation formal charge of +3 to Bader charges ranging from 1.85 to 2.20 (see Table S1). Accordingly, our simulations show that supported $\left[\mathrm{MoO}(\mathrm{OH})_{3}\right]^{-}$clusters containing $\mathrm{Mo}^{4+}$ are the most stable species around the RHE potential (Figure 4). However, below $-0.23 \mathrm{~V}$ vs RHE, the highest thermodynamic stability is shown by $\left[\mathrm{H}_{2} \mathrm{Mo}(\mathrm{OH})_{2}\right]^{-}$and $\left[\mathrm{H}_{3} \mathrm{MoOH}\right]^{-}$with Mo cations in a $3+$ oxidation state. The increased stability of the clusters containing $\mathrm{Mo}^{3+}$ and $\mathrm{Mo}^{4+}$ is partially due to the electron transfer from the $\mathrm{Ni}(111)$ support to the clusters and the presence of direct $\mathrm{Ni}-\mathrm{Mo}$ bonds, which are absent in more oxidized Mo clusters (Table S3). Thus, our simulations corroborate the experimental findings that $\mathrm{Mo}^{3+}$ centers can form on $\mathrm{Ni}_{x} \mathrm{Mo}_{y}$ catalysts under HER conditions. Moreover, $\mathrm{Mo}^{3+}$ centers in supported $\left[\mathrm{H}_{2} \mathrm{Mo}(\mathrm{OH})_{2}\right]^{-}$and $\left[\mathrm{H}_{3} \mathrm{MoOH}\right]^{-}$ clusters have magnetization values of 0.9 and $1.3 \mu_{\mathrm{B}}$, respectively, in line with the appearance of a $\mathrm{Mo}^{3+}$ signal in EPR experiments.

Finally, calculated HER overpotentials were compared on supported $\mathrm{H}_{x} \mathrm{MoO}_{y}(\mathrm{OH})_{z}$ clusters, a Ni-Mo alloy, and pure $\mathrm{Ni}$ nanoparticles, through a combination of alkaline Volmer and alkaline Heyrovsky steps (experimentally obtained Tafel slope of $\sim 100 \mathrm{mV} \mathrm{dec}{ }^{-1}$ at $-0.12 \mathrm{~V}$ vs RHE). For supported Mo species, we calculated reaction energy profiles for two forms of the HER mechanism (Figure S10)

$$
\mathrm{MoO}_{m} \mathrm{H}_{n} \stackrel{+\mathrm{H}_{2} \mathrm{O}+\mathrm{e}^{-}-\mathrm{OH}^{-}}{\longrightarrow} \mathrm{MoO}_{m} \mathrm{H}_{n+1} \stackrel{+\mathrm{H}_{2} \mathrm{O}+\mathrm{e}^{-}-\mathrm{OH}^{-}-\mathrm{H}_{2}}{\longrightarrow} \mathrm{MoO}_{m} \mathrm{H}_{n}
$$

or

$$
\mathrm{MoO}_{m} \mathrm{H}_{n} \stackrel{+\mathrm{H}_{2} \mathrm{O}+\mathrm{e}^{-}-\mathrm{OH}^{-}-\mathrm{H}_{2}}{\longrightarrow} \mathrm{MoO}_{m} \mathrm{H}_{n-1} \stackrel{+\mathrm{H}_{2} \mathrm{O}+\mathrm{e}^{-}-\mathrm{OH}^{-}}{\longrightarrow} \mathrm{MoO}_{m} \mathrm{H}_{n}
$$

The clusters containing $\mathrm{Mo}^{3+}$ centers were calculated to have less significant HER overpotentials than those of the pure $\mathrm{Ni}$ and $\mathrm{Ni}_{x} \mathrm{Mo}_{y}$ alloy $(\sim 0.38$ and $\sim 0.43 \mathrm{~V}$, respectively) and to have high thermodynamic stability at electrode potentials below RHE (see Figure 5 and Table S4 for details). In particular, $\mathrm{Mo}^{3+}$ centers in $\mathrm{H}_{2} \mathrm{Mo}(\mathrm{OH})_{2}$ catalyze HER with an overpotential of only $0.05 \mathrm{~V}$, which is much smaller than HER overpotentials on more oxidized clusters. At lower electrode potentials, $\mathrm{Mo}^{3+}$ centers in $\left[\mathrm{H}_{3} \mathrm{MoOH}\right]^{-}$clusters supported on $\mathrm{Ni}(111)$ can catalyze the critical water-splitting step in the HER catalytic cycle with an overpotential of just $0.24 \mathrm{~V}$. Hence, our calculations demonstrate that $\mathrm{Mo}^{3+}$ centers can be thermodynamically stable on a $\mathrm{Ni}(111)$ surface under HER conditions and that they can catalyze HER at a much lower overpotential than pure $\mathrm{Ni}$ or $\mathrm{Ni}_{x} \mathrm{Mo}_{y}$ alloy. Note that the 
considered HER mechanisms on Mo clusters do not involve $\mathrm{H}$ or $\mathrm{OH}$ species bound to the $\mathrm{Ni}$ surface, which may explain the much lower effect of $\mathrm{CO}$ poisoning on the HER activity of $\mathrm{Ni}-\mathrm{Mo}$ catalysts, compared to pure Ni.

\section{CONCLUSIONS}

In conclusion, in situ spectroscopic characterization, electrochemical measurements, and DFT calculations demonstrated that a Mo oxo species (most likely $\mathrm{Mo}^{3+}$ ) supported on metallic $\mathrm{Ni}$ or $\mathrm{Ni}_{x} \mathrm{Mo}_{y}$ alloy was responsible for the high activity of a Ni-Mo catalyst for water reduction to form $\mathrm{H}_{2}$. Although oxidized Mo species were present already in an opencircuit state as evident from Raman spectroscopy, the active Mo oxo species for HER was formed upon applying the HER potentials. Metallic $\mathrm{Ni}$ or $\mathrm{Ni}_{x} \mathrm{Mo}_{y}$ underneath would serve as a conductive anchor to the oxidized Mo species, preventing dissolution and modifying the electronic structure of the Mo surface species. The illustration of such a mechanism highlights the critical ability of Mo oxo species to carry out water reduction as an earth-abundant catalyst and the influence of support on its activity. These results may be extendable to the large family of Mo-based HER catalysts that have been reported in the past decade. Our findings also illustrate the behavior of a metal oxide (molybdenum oxide) interacting with a metal $(\mathrm{Ni})$ and its importance for the overall catalytic activity.

\section{ASSOCIATED CONTENT}

\section{SI Supporting Information}

The Supporting Information is available free of charge at https://pubs.acs.org/doi/10.1021/acscatal.0c02743.

Experimental and computational methods and supplementary data (Figures S1-S10 and Tables S1-S4) (PDF)

\section{AUTHOR INFORMATION}

\section{Corresponding Authors}

Luigi Cavallo - King Abdullah University of Science and Technology (KAUST), KAUST Catalysis Center (KCC), Thuwal 23955-6900, Saudi Arabia; orcid.org/0000-00021398-338X; Email: luigi.cavallo@kaust.edu.sa

Kazuhiro Takanabe - King Abdullah University of Science and Technology (KAUST), KAUST Catalysis Center (KCC), Thuwal 23955-6900, Saudi Arabia; Department of Chemical System Engineering, School of Engineering, The University of Tokyo, Tokyo 113-8656, Japan; 이이이.org/0000-00015374-9451; Email: takanabe@chemsys.t.u-tokyo.ac.jp

\section{Authors}

Jeremy A. Bau - King Abdullah University of Science and Technology (KAUST), KAUST Catalysis Center (KCC), Thuwal 23955-6900, Saudi Arabia; 이이이.org/0000-00028264-0698

Sergey M. Kozlov - King Abdullah University of Science and Technology (KAUST), KAUST Catalysis Center (KCC), Thuwal 23955-6900, Saudi Arabia; Department of Chemical and Biomolecular Engineering, National University of Singapore, Singapore 117585

Luis Miguel Azofra - King Abdullah University of Science and Technology (KAUST), KAUST Catalysis Center (KCC), Thuwal 23955-6900, Saudi Arabia; Instituto de Estudios Ambientales y Recursos Naturales (i-UNAT), Universidad de
Las Palmas de Gran Canaria (ULPGC), 35017 Las Palmas de Gran Canaria, Spain; 이이.org/0000-0003-4974-1670

Samy Ould-Chikh - King Abdullah University of Science and Technology (KAUST), KAUST Catalysis Center (KCC), Thuwal 23955-6900, Saudi Arabia; 이이.org/0000-00023486-0944

Abdul-Hamid Emwas - King Abdullah University of Science and Technology (KAUST), KAUST Imaging and Characterization Core Lab, Thuwal 23955-6900, Saudi Arabia

Hicham Idriss - Centre for Research and Development, Saudi Arabian Basic Industries Corporation (SABIC), Thuwal 23955-6900, Saudi Arabia; 이 orcid.org/0000-0001-86147019

Complete contact information is available at:

https://pubs.acs.org/10.1021/acscatal.0c02743

\section{Author Contributions}

$\nabla$ J.A.B and S.M.K. made equal contributions as co-first authors. Notes

The authors declare no competing financial interest.

\section{ACKNOWLEDGMENTS}

The research reported in this study was supported by the King Abdullah University of Science and Technology. The authors acknowledge the support of SABIC in funding this research. Computational resources were provided primarily by the KAUST Supercomputing Laboratory and the Shaheen II supercomputer. Computational work was also partially performed using resources of the National Supercomputing Centre, Singapore (https://www.nscc.sg).

\section{REFERENCES}

(1) Bard, A. J.; Fox, M. A. Artificial Photosynthesis: Solar Splitting of Water to Hydrogen and Oxygen. Acc. Chem. Res. 1995, 28, 141-145.

(2) Roger, I.; Shipman, M. A.; Symes, M. D. Earth-Abundant Catalysts for Electrochemical and Photoelectrochemical Water Splitting. Nat. Rev. Chem. 2017, 1, No. 0003.

(3) Montoya, J. H.; Seitz, L. C.; Chakthranont, P.; Vojvodic, A.; Jaramillo, T. F.; Nørskov, J. K. Materials for Solar Fuels and Chemicals. Nat. Mater. 2017, 16, 70-81.

(4) Wang, T.; Xie, H.; Chen, M.; D’Aloia, A.; Cho, J.; Wu, G.; Li, Q. Precious Metal-Free Approach to Hydrogen Electrocatalysis for Energy Conversion: From Mechanism Understanding to Catalyst Design. Nano Energy 2017, 42, 69-89.

(5) Sheng, W.; Gasteiger, H. A.; Shao-Horn, Y. Hydrogen Oxidation and Evolution Reaction Kineticsh on Platinum: Acid vs Alkaline Electrolytes. J. Electrochem. Soc. 2010, 157, B1529-B1536.

(6) Shinagawa, T.; Takanabe, K. Identification of intrinsic catalytic activity for electrochemical reduction of water molecules to generate hydrogen. Phys. Chem. Chem. Phys. 2015, 17, 15111-15114.

(7) Subbaraman, R.; Tripkovic, D.; Strmcnik, D.; Chang, K.-C.; Uchimura, M.; Paulikas, A. P.; Stamenkovic, V.; Markovic, N. M. Enhancing Hydrogen Evolution Activity in Water Splitting by Tailoring Li+-Ni(OH) $)_{2}$-Pt Interfaces. Science 2011, 334, 1256-1260.

(8) Gong, M.; Zhou, W.; Tsai, M.-C.; Zhou, J.; Guan, M.; Lin, M.C.; Zhang, B.; Hu, Y.; Wang, D.-Y.; Yang, J.; Pennycook, S. J.; Hwang, B.-J.; Dai, H. Nanoscale Nickel Oxide/Nickel Heterostructures for Active Hydrogen Evolution Electrocatalysis. Nat. Commun. 2014, 5, No. 4695.

(9) Wang, G.; Parrondo, J.; He, C.; Li, Y.; Ramani, V. Pt/C/ $\mathrm{Ni}(\mathrm{OH})_{2}$ Bi-Functional Electrocatalyst for Enhanced Hydrogen Evolution Reaction Activity under Alkaline Conditions. J. Electrochem. Soc. 2017, 164, F1307-F1315.

(10) Ledezma-Yanez, I.; Wallace, W. D. Z.; Sebastián-Pascual, P.; Climent, V.; Feliu, J. M.; Koper, M. T. M. Interfacial Water 
Reorganization as a pH-Dependent Descriptor of the Hydrogen Evolution Rate on Platinum Electrodes. Nat. Energy 2017, 2, No. 17031.

(11) Mahmood, N.; Yao, Y.; Zhang, J.-W.; Pan, L.; Zhang, X.; Zou, J.-J. Electrocatalysts for Hydrogen Evolution in Alkaline Electrolytes: Mechanisms, Challenges, and Prospective Solutions. Adv. Sci. 2017, 5, No. 1700464.

(12) Strmcnik, D.; Lopes, P. P.; Genorio, B.; Stamenkovic, V. R.; Markovic, N. M. Design Principles for Hydrogen Evolution Reaction Catalyst Materials. Nano Energy 2016, 29, 29-36.

(13) McCrory, C. C. L.; Jung, S.; Ferrer, I. M.; Chatman, S. M.; Peters, J. C.; Jaramillo, T. F. Benchmarking Hydrogen Evolving Reaction and Oxygen Evolving Reaction Electrocatalysts for Solar Water Splitting Devices. J. Am. Chem. Soc. 2015, 137, 4347-4357.

(14) Zhang, J.; Wang, T.; Liu, P.; Liao, Z.; Liu, S.; Zhuang, X.; Chen, M.; Zschech, E.; Feng, X. Efficient Hydrogen Production on $\mathrm{MoNi}_{4}$ Electrocatalysts with Fast Water Dissociation Kinetics. Nat. Commun. 2017, 8, No. 15437.

(15) Wang, Y.; Zhang, G.; Xu, W.; Wan, P.; Lu, Z.; Li, Y.; Sun, X. A 3D Nanoporous Ni-Mo Electrocatalyst with Negligible Overpotential for Alkaline Hydrogen Evolution. ChemElectroChem 2014, $1,1138-1144$.

(16) Soriaga, M. P.; Baricuatro, J. H.; Cummins, K. D.; Kim, Y.-G.; Saadi, F. H.; Sun, G.; McCrory, C. C. L.; McKone, J. R.; Velazquez, J. M.; Ferrer, I. M.; Carim, A. I.; Javier, A.; Chmielowiec, B.; Lacy, D. C.; Gregoire, J. M.; Sanabria-Chinchilla, J.; Amashukeli, X.; Royea, W. J.; Brunschwig, B. S.; Hemminger, J. C.; Lewis, N. S.; Stickney, J. L. Electrochemical Surface Science Twenty Years Later: Expeditions into the Electrocatalysis of Reactions at the Core of Artificial Photosynthesis. Surf. Sci. 2015, 631, 285-294.

(17) McKone, J. R.; Marinescu, S. C.; Brunschwig, B. S.; Winkler, J. R.; Gray, H. B. Earth-Abundant Hydrogen Evolution Electrocatalysts. Chem. Sci. 2014, 5, 865-878.

(18) Kawashima, A.; Akiyama, E.; Habazaki, H.; Hashimoto, K. Characterization of Sputter-Deposited Ni-Mo and Ni-W Alloy Electrocatalysts for Hydrogen Evolution in Alkaline Solution. Mater. Sci. Eng., A 1997, 226-228, 905-909.

(19) Schalenbach, M.; Speck, F. D.; Ledendecker, M.; Kasian, O.; Goehl, D.; Mingers, A. M.; Breitbach, B.; Springer, H.; Cherevko, S.; Mayrhofer, K. J. J. Nickel-Molybdenum Alloy Catalysts for the Hydrogen Evolution Reaction: Activity and Stability Revised. Electrochim. Acta 2018, 259, 1154-1161.

(20) Navarro-Flores, E.; Chong, Z.; Omanovic, S. Characterization of $\mathrm{Ni}, \mathrm{NiMo}, \mathrm{NiW}$ and $\mathrm{NiFe}$ Electroactive Coatings as Electrocatalysts for Hydrogen Evolution in an Acidic Medium. J. Mol. Catal. A: Chem. 2005, 226, 179-197.

(21) Jakšić, J. M.; Vojnović, M. V.; Krstajić, N. V. Kinetic Analysis of Hydrogen Evolution at Ni-Mo Alloy Electrodes. Electrochim. Acta 2000, 45, 4151-4158.

(22) Highfield, J. G.; Claude, E.; Oguro, K. Electrocatalytic Synergism in Ni/Mo Cathodes for Hydrogen Evolution in Acid Medium: A New Model. Electrochim. Acta 1999, 44, 2805-2814.

(23) Liu, X.; Ni, K.; Niu, C.; Guo, R.; Xi, W.; Wang, Z.; Meng, J.; Li, J.; Zhu, Y.; Wu, P.; Li, Q.; Wu, X.; Mai, L.; et al. Upraising the O $2 p$ Orbital by Integrating $\mathrm{Ni}$ with $\mathrm{MoO}_{2}$ for Accelerating Hydrogen Evolution Kinetics. ACS Catal. 2019, 9, 2275-2285.

(24) Karunadasa, H. I.; Chang, C. J.; Long, J. R. A Molecular Molybdenum-Oxo Catalyst for Generating Hydrogen from Water. Nature 2010, 464, 1329-1333.

(25) Li, J.; Yoshizawa, K. Computational Evidence for Hydrogen Generation by Reductive Cleavage of Water and $\alpha-\mathrm{H}$ Abstraction on a Molybdenum Complex. Angew. Chem., Int. Ed. 2011, 50, 1197211975.

(26) Prior, C.; Webster, L. R.; Ibrahim, S. K.; Wright, J. A.; Alghamdi, A. F.; Oganesyan, V. S.; Pickett, C. J. EPR Detection and Characterisation of a Paramagnetic Mo(III) Dihydride Intermediate Involved in Electrocatalytic Hydrogen Evolution. Dalton Trans. 2016, $45,2399-2403$
(27) Jin, Y.; Wang, H.; Li, J.; Yue, X.; Han, Y.; Shen, P.-K.; Cui, Y. Porous $\mathrm{MoO}_{2}$ Nanosheets as Non-noble Bifunctional Electrocatalysts for Overall Water Splitting. Adv. Mater. 2016, 28, 3785-3790.

(28) Deng, Y.; Yeo, B. S. Characterization of Electrocatalytic Water Splitting and $\mathrm{CO}_{2}$ Reduction Reactions Using In Situ/Operando Raman Spectroscopy. ACS Catal. 2017, 7, 7873-7889.

(29) Gao, P.; Gosztola, D.; Leung, L.-W. H.; Weaver, M. J. SurfaceEnhanced Raman Scattering at Gold Electrodes: Dependence on Electrochemical Pretreatment Conditions and Comparisons with Silver. J. Electroanal. Chem. Interfacial Electrochem. 1987, 233, 211222.

(30) Kresse, G.; Furthmüller, J. Efficient Iterative Schemes for Ab Initio Total-Energy Calculations Using a Plane-Wave Basis Set. Phys. Rev. B 1996, 54, 11169-11186.

(31) Hammer, B.; Hansen, L. B.; Nørskov, J. K. Improved Adsorption Energetics within Density-Functional Theory using Revised Perdew-Burke-Ernzerhof Functionals. Phys. Rev. B 1999, 59, $7413-7421$.

(32) Kresse, G.; Joubert, D. From Ultrasoft Pseudopotentials to the Projector Augmented-Wave Method. Phys. Rev. B 1999, 59, 17581775.

(33) Dudarev, S. L.; Botton, G. A.; Savrasov, S. Y.; Humphreys, C. J.; Sutton, A. P. Electron- energy-loss spectra and the structural stability of nickel oxide: An LSDA+U study. Phys. Rev. B 1998, 57, 15051509.

(34) Grimme, S.; Antony, J.; Ehrlich, S.; Krieg, H. A consistent and accurate $\mathrm{ab}$ initio parametrization of density functional dispersion correction (DFT-D) for the 94 elements H-Pu. J. Chem. Phys. 2010, 132, No. 154104.

(35) Mathew, K.; Sundararaman, R.; Letchworth-Weaver, K.; Arias, T. A.; Hennig, R. G. Implicit solvation model for density-functional study of nanocrystal surfaces and reaction pathways. J. Chem. Phys. 2014, 140, No. 084106.

(36) Vu, Q. K.; Chassaing, E.; Bourelier, F.; Montuelle, J. M. Uncracked Electrolytic Ni-Mo Alloy Plating and Heat Treatments to Improve the Acid Corrosion Resistance. Corros.-Trait., Prot. 1971, 19, 237-248.

(37) McKone, J. R.; Sadtler, B. F.; Werlang, C. A.; Lewis, N. S.; Gray, H. B. Ni-Mo Nanopowders for Efficient Electrochemical Hydrogen Evolution. ACS Catal. 2013, 3, 166-169.

(38) Soriaga, M. P.; Baricuatro, J. H.; Cummins, K. D.; Kim, Y.-G.; Saadi, F. H.; Sun, G.; McCrory, C. C. L.; McKone, J. R.; Velazquez, J. M.; Ferrer, I. M.; Carim, A. I.; Javier, A.; Chmielowiec, B.; Lacy, D. C.; Gregoire, J. M.; Sanabria-Chinchilla, J.; Amashukeli, X.; Royea, W. J.; Brunschwig, B. S.; Hemminger, J. C.; Lewis, N. S.; Stickney, J. L. Electrochemical surface science twenty years later: Expeditions into the electrocatalysis of reactions at the core of artificial photosynthesis. Surf. Sci. 2015, 631, 285-294.

(39) Dmochowska, M.; Czerwiński, A. Behavior of a Nickel Electrode in the Presence of Carbon Monoxide. J Solid State Electrochem. 1998, 2, 16-23.

(40) Hori, Y.; Murata, A. Electrochemical Evidence of Intermediate Formation of Adsorbed $\mathrm{CO}$ in Cathodic Reduction of $\mathrm{CO}_{2}$ at a Nickel Electrode. Electrochim. Acta 1990, 35, 1777-1780.

(41) Strmcnik, D.; Kodama, K.; van der Vliet, D.; Greeley, J.; Stamenkovic, V. R.; Marković, N. M. The Role of Non-Covalent Interactions in Electrocatalytic Fuel-Cell Reactions on Platinum. Nat. Chem. 2009, 1, 466-472.

(42) Csernica, P. M.; McKone, J. R.; Mulzer, C. R.; Dichtel, W. R.; Abruña, H. D.; DiSalvo, F. J. Electrochemical Hydrogen Evolution at Ordered $\mathrm{Mo}_{7} \mathrm{Ni}_{7}$. ACS Catal. 2017, 7, 3375-3383.

(43) Hall, D. S.; Bock, C.; MacDougall, B. R. The Electrochemistry of Metallic Nickel: Oxides, Hydroxides, Hydrides and Alkaline Hydrogen Evolution. J. Electrochem. Soc. 2013, 160, F235-F243.

(44) Hardcastle, F. D.; Wachs, I. E. Determination of molybdenumoxygen bond distances and bond orders by Raman spectroscopy. J. Raman Spectrosc. 1990, 21, 683-691.

(45) Bau, J.; Haspel, H.; Ould-Chikh, S.; Tapia, A. A.; Hazemann, J.L.; Idriss, H.; Takanabe, K. On the Reconstruction of NiMo 
Electrocatalysts by Operando Spectroscopy. J. Mater. Chem. A 2019, 7, 15031-15035.

(46) Hunter, B. M.; Thompson, N. B.; Müller, A. M.; Rossman, G. R.; Hill, M. G.; Winkler, J. R.; Gray, H. B. Trapping an Iron(VI) Water-Splitting Intermediate in Nonaqueous Media. Joule 2018, 2, $747-763$.

(47) Dubouis, N.; Serva, A.; Salager, E.; Deschamps, M.; Salanne, M.; Grimaud, A. The Fate of Water at the Electrochemical Interfaces: Electrochemical Behavior of Free Water Versus Coordinating Water. J. Phys. Chem. Lett. 2018, 9, 6683-6688.

(48) Mann, R. S.; Khulbe, K. C. ESR Study of $\mathrm{MoO}_{3}$ Obtained from Thermal Decomposition of Ammonium Molybdate. Bull. Chem. Soc. Jpn. 1975, 48, 1021-1023.

(49) Radhakrishna, S.; Chowdari, B. V. R.; Viswanath, A. K. EPR Studies of $\mathrm{Mo}^{3+}$ Ion in Single Crystals of $\mathrm{NH}_{4} \mathrm{Al}\left(\mathrm{SO}_{4}\right)_{2} \cdot 12 \mathrm{H}_{2} \mathrm{O}$. J. Chem. Phys. 1977, 66, 2009-2014.

(50) Chiesa, M.; Giamello, E.; Che, M. EPR Characterization and Reactivity of Surface-Localized Inorganic Radicals and Radical Ions. Chem. Rev. 2010, 110, 1320-1347.

(51) Villacampa, B.; Alcalá, R.; Alonso, P. J.; et al. EPR study of Ni+ centers in $\mathrm{CsCaF}_{3}$. Phys. Rev. B 1994, 49, 1039-1047.

(52) Reuter, K. Ab Initio Thermodynamics and First-Principles Microkinetics for Surface Catalysis. In Operando Research in Heterogeneous Catalysis; Frenken, J.; Groot, I., Eds.; Springer Series in Chemical Physics; Springer International Publishing: Cham, 2017; pp 151-188.

(53) Walsh, A.; Sokol, A. A.; Buckeridge, J.; Scanlon, D. O.; Catlow, C. R. A. Electron Counting in Solids: Oxidation States, Partial Charges, and Ionicity. J. Phys. Chem. Lett. 2017, 8, 2074-2075. 